EPJ Web of Conferences 41, 09016 (2013)

DOI: $10.1051 /$ epjconf/20134109016

(C) Owned by the authors, published by EDP Sciences, 2013

\title{
Photomixing for Coherent Retrieval of THz Waveforms from a Frequency Multiplier
}

\author{
F. L. Constantin ${ }^{1}$ \\ ${ }^{1}$ Laboratoire PhLAM, UMR 8523, 59655 Villeneuve d'Ascq, France
}

\begin{abstract}
THz}$ waveforms generated with an electronic frequency multiplier are sampled with a heterodyne detection technique using a LTG-GaAs photomixer and the optical beat of two near-infrared lasers.
\end{abstract}

\section{Introduction}

The optical heterodyne conversion (photomixing) is a well-established approach used for bridging the terahertz spectral domain. This technique relies on a photoconductor coupled through a planar antenna (photomixer) with a response time in the sub-ps range. Two cw lasers emitting above the photoconductor bandgap are frequency detuned by a THz gap and irradiates the device. Upon the photomixer is biased, the photocurrent oscillating at the difference frequency drives the antenna and generates $\mathrm{THz}$ waves [1]. The photomixer used in this work is an LTG-GaAs photoconductor with a pattern of interdigitated electrodes driven by a spiral self-complementary antenna coupled to a 50ohm microwave line. It has a quadratic response to the optical fields and an intrinsic non-linear current-voltage dependence. These characteristics have been exploited in direct and heterodyne detection schemes with the photomixer [2]. This contribution demonstrates two asynchronous optical sampling schemes with of the photomixing setup for terahertz pulse measurements.

\section{Experimental setup}

The THz source used in the experiment (Fig. 1.A) is an electronic frequency multiplier (Millitech AMC-10-R0000) driven by a microwave synthesizer (MW). It multiplies 6 times the MW signal and provides $\sim 2 \mathrm{~mW} \mathrm{cw}$ signal output in $75-110 \mathrm{GHz}$ range. Terahertz waveforms are generated by pulse-modulating the MW synthesizer with a $\mathrm{RF}$ synthesiser $\left(\mathrm{RF}_{\mathrm{THz}}\right)$. The pulse repetition frequency and the $\mathrm{THz}$ carrier frequency are phase-referenced to the quartz oscillator. The THz source output is focused on the antenna with a silicon lens mounted against the photomixer chip. The photomixer is driven by two extended-cavity diode lasers (DL1, DL2) emitting around $825 \mathrm{~nm}$ operated in a freerunning mode. The laser beams are spatially overlapped, frequency shifted with an acousto-optic modulator (AOM) and focused on the photomixer electrodes. Laser beams can be pulse-modulated by switching the $\mathrm{AOM}$ with a $\mathrm{RF}$ synthesiser $\left(\mathrm{RF}_{\mathrm{AOM}}\right)$ referenced to the quartz oscillator. That allows to have the same time reference for the pulsed operation of the optical and the $\mathrm{THz}$ source. Microwave (AC port) and low-frequency (DC port) responses of the photomixer are addressed through a Bias-T and measured with a spectrum analyser (SA) with 50- $\Omega$ input impedance.

This is an Open Access article distributed under the terms of the Creative Commons Attribution License 2.0, which permits unrestricted use, distribution, and reproduction in any medium, provided the original work is properly cited. 

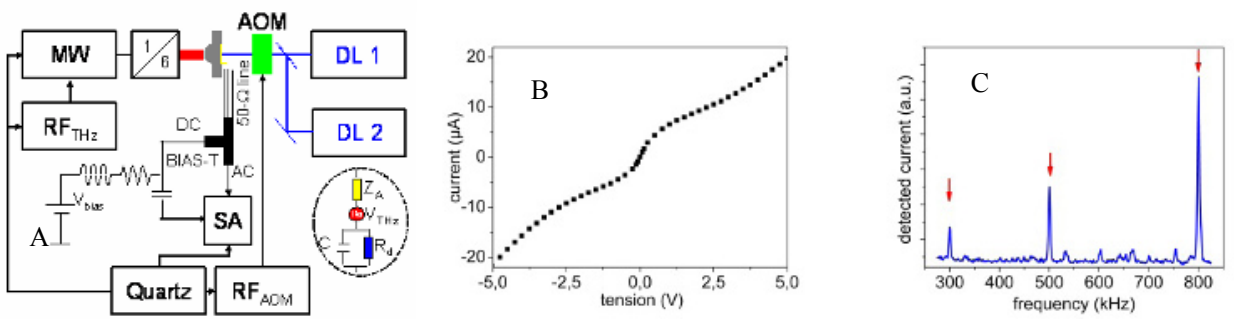

Fig. 1.A. Experimental setup. The photomixer is biased through a RL choke. SA is alternatively coupled on the DC port or capacitively on the AC port. Inset: equivalent model circuit for the detection with the photomixer. $\mathrm{R}_{\mathrm{d}}$ dynamic resistance at the bias point under the given optical power, $\mathrm{V}_{\mathrm{THz}}$ antennainduced Thévenin voltage, $Z_{\mathrm{A}}$ antenna impedance. $Z_{\mathrm{A}}=72-\Omega$ at THz frequencies, it has a complex dependence in the microwave regime and the obvious limit $Z A \rightarrow 0$ at DC.

Fig. 1.B. Current-voltage dependence of the photomixer under $8 \mathrm{~mW}$ optical power.

Fig. 1.C. ASOPS with the pulsed laser source. Optical power $16 \mathrm{~mW}$, bias $-18 \mathrm{mV}$. RBW=3 kHz, sweep time $152.8 \mathrm{~ms}, 50$ video averages

\section{Terahertz waveform sampling with laser pulse-modulation}

Detection with the photomixer can be described in terms of rectification in the small-signal regime. The THz wave incident on the antenna induces a THz Thévenin voltage in the photomixer circuit, depending on a coupling factor, the $\mathrm{THz}$ electric field and the antenna impedance. The rectified current depends on the second-order derivative of the current-voltage characteristic (Fig 1. B). In addition, the $\mathrm{THz}$ voltage applied on the optically-modulated conductance of the photomixer allows sampling the terahertz field amplitude with the laser pulses. An asynchronous optical sampling (ASOPS) scheme is demonstrated by pulse-modulating the laser source and the THz source at slight different repetition rates (respectively at $800 \mathrm{kHz}$ and $500 \mathrm{kHz}$ ). The frequency-domain dependence of the low-frequency photomixer current (Fig. 1.C) displays the laser pulsed-modulation signal, the $\mathrm{THz}$ rectification signal as well as the ASOPS signal at $300 \mathrm{kHz}$, corresponding to the difference of the pulse-modulation frequencies.

\section{Terahertz waveform sampling with pulse-modulation of the optical beat}

In the heterodyne detection, the antenna-induced Thévenin voltage is applied on the conductance modulated by the optical beat. That leads to a down-converted signal in the microwave domain. This can be discussed in terms of rectification in the small-signal regime. The optical beat-induced voltage mixes with the antenna-induced voltage through the photomixer's second-order currentvoltage nonlinearity. That yields the heterodyne current carrier at the difference-frequency between the optical beat and the terahertz source. Using a pulsed optical beat and a pulsed $\mathrm{THz}$ source leads to multi-frequency heterodyne mixing which adds sidebands to the carrier that are spaced by the pulse modulation frequencies.

$\mathrm{THz}$ source operated at $86 \mathrm{GHz}$ is pulse-modulated at $9 \mathrm{MHz}$. The resulting frequency comb is down-converted to the microwave domain with the optical beat. The current spectrum recorded in the microwave range (Fig 2.A) with the non-modulated optical beat displays sidebands separated by the pulse repetition frequency over $\sim 150 \mathrm{MHz}$ frequency span. Alternatively, the non-modulated $\mathrm{THz}$ source is down-converted with an optical beat that is pulse-modulated with the AOM at 2.2 MHz. The current spectrum (Fig. 2.B) displays sidebands separated by the pulse-repetition frequency with $\sim 1 \mathrm{MHz}$ linewidth determined by the spectral purity of the free-running diode lasers. When both $\mathrm{THz}$ source and optical beat are pulse-modulated (respectively at $9 \mathrm{MHz}$ and $2.2 \mathrm{MHz}$ ) a multifrequency heterodyne asynchronous optical sampling is demonstrated. Fig. 2.C displays the current 
spectrum where the most intense spectral features are separated by the pulse-repetition frequency of the $\mathrm{THz}$ source. Each spectral feature has a structure shown in the inset with sidebands separated by the AOM pulse-modulation frequency that is similar with spectrum displayed in Fig. 2.B, although the SNR is smaller. In addition, a part of the current spectrum generated in the photomixer by the pulsed optical beat and the pulsed $\mathrm{THz}$ source has been recorded at low-frequency (Fig. 2.D). It displays the $\mathrm{THz}$ rectification signal at $9 \mathrm{MHz}$ as well as th e multi-heterodyne mixing sidebands respectively at $6.8 \mathrm{MHz}$ and $11.2 \mathrm{MHz}$.
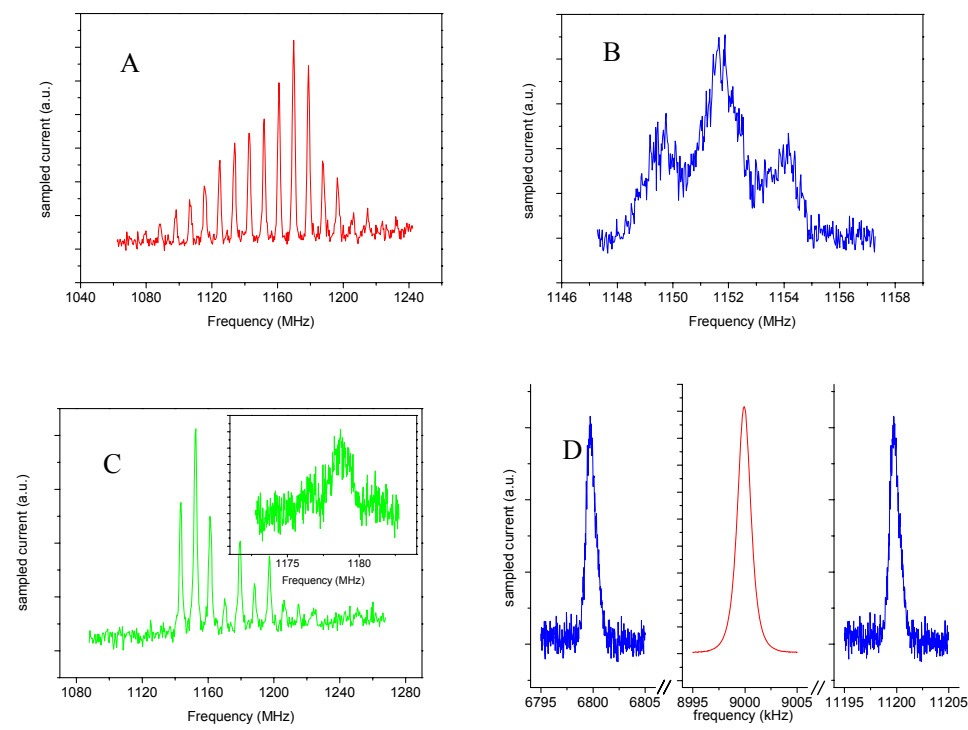

Fig. 2.A. Frequency dependence of the THz comb. Bias $52 \mathrm{mV}$, combined optical power $16 \mathrm{~mW}$. $\mathrm{RBW}=1 \mathrm{MHz}$, sweep time $4 \mathrm{~ms}$, 50 video averages.

Fig. 2.B. Frequency dependence of the optical beat comb. Bias $52 \mathrm{mV}$, combined optical power $16 \mathrm{~mW}$. $\mathrm{RBW}=100 \mathrm{kHz}$, sweep time $5 \mathrm{~ms}, 50$ video averages.

Fig. 2.C. ASOPS with the pulsed optical beat. Bias $52 \mathrm{mV}$, combined optical power $16 \mathrm{~mW}$. RBW=1 $\mathrm{MHz}$, sweep time $4 \mathrm{~ms}, 50$ video averages. Inset: a component of the frequency comb, RBW=100 kHz, sweep time $5 \mathrm{~ms}, 50$ video averages.

Fig. 2.D. Low-frequency sampled signals. $\mathrm{RBW}=1 \mathrm{kHz}$, sweep time $275 \mathrm{~ms}, 100$ video averages.

\section{Conclusion}

Asynchronous optical sampling of a pulsed $\mathrm{THz}$ electronic frequency multiplier with a photomixer is demonstrated. This approach has the advantage to deliver in a straightforward way $\mathrm{THz}$ pulses with carrier frequency and pulse-repetition rate referenced to a frequency standard. The exceptional frequency bandwidth of the photomixer opens the way to characterize with the ASOPS scheme the pulsed operation of electronic multipliers over a broad spectral domain.

\section{References}

1. E. R. Brown, F. W. Smith, and K. A. MacIntosh, J. Appl. Phys. 73, 1480 (1993)

2. F. L. Constantin, IEEE J. Quantum Electr. 47, 1458 (2011) 